\title{
Correspondence
}

\section{Sampled-Data Filtering with Error Covariance Assignment}

Zidong Wang, Biao Huang, and Peijun Huo

\begin{abstract}
In this correspondence, we consider the sampled-data filtering problem by proposing a new performance criterion in terms of the estimation error covariance. An innovation approach to sampled-data filtering is presented. First, the definition of the estimation covariance for a sampled-data system is given, then the sampled-data filtering problem is reduced to the Kalman filter design problem for a fictitious discrete-time system, and finally, an effective method is developed to design discrete-time Kalman filters in such a way that the resulting sampled-data estimation covariance achieves a prescribed value. We derive both the existence conditions and the explicit expression of the desired filters and provide an illustrative numerical example to demonstrate the directness and flexibility of the present design method.
\end{abstract}

Index Terms-Continuous-time systems, discrete-time systems, Kalman filtering, sampled-date filtering, intersample behavior.

\section{INTRODUCTION}

Owing to the advances in digital computers, discrete-time filtering of continuous-time systems has been developed and used in numerous applications, and thus, optimal sampled-data filter design problem has well been studied; see, e.g., [2] and references therein. The aim of the optimal sampled-data filtering problem is, for continuous-time systems with continuous-time measurements, to design discrete-time filter that, with A/D averaged measurements and D/A zero-order-hold estimates, minimizes the least squares estimation criterion. The optimal sampled-data filtering approach, however, is not suitable for the case when the performance objectives are expressed explicitly in terms of the steady-state estimation error variance, that is, the estimation error variance is not necessarily minimal but should meet certain upper bound constraints. This case is quite common in practical filtering problems, such as tracking of maneuvering target and recognition of flight paths from multiple sources [5]. Traditional optimal filtering theories could minimize a selected weighted scalar sum of the error variances of the state estimation in order to indirectly achieve the steady-state error variance constraints, but minimizing a scalar sum does not ensure that the multiple variance requirements will be satisfied.

Recently, a novel filtering method, namely, error covariance assignment (ECA) theory (see, e.g., [14] and [15]) was developed to provide an alternative and more straightforward methodology for designing filter gains that satisfy the error covariance constraints. This methodology could provide a closed-form solution for directly assigning the

Manuscript received February 9, 1999; revised October 11, 2000. This work was supported by the Alexander von Humboldt Foundation of Germany, the University of Kaiserslautern, and the National Natural Science Foundation of China. The associate editor coordinating the review of this paper and approving it for publication was Dr. Brian M. Sadler.

Z. Wang is with the Department of Mathematics, University of Kaiserslautern, Kaiserslautern, Germany, on leave from the Department of Automatic Control, Nanjing University of Science and Technology, Nanjing, China. He is also with the Control Theory and Applications Center, School of Mathematical and Information Sciences, Coventry University, Coventry, U.K.

B. Huang is with the Department of Chemical and Materials Engineering, University of Alberta, Edmonton, AB, Canada T6G 2G6.

P. Huo is with the Department of Management Engineering, Shanghai Jiao Tong University, Shanghai, China.

Publisher Item Identifier S 1053-587X(01)00628-6. specified steady-state estimation error covariance, and thus, the desired error variance constraints could be achieved. With the ECA theory, we are able to parameterize all filters/estimators in terms of the performance criterion of the state estimation error covariance that is physically meaningful in practice and to deal with the system noise or output noise directly. Subsequently, [9], [10], [12], and [13], extended the ECA theory to the parameter uncertain systems by assigning a prescribed upper bound to the steady-state error variance. The purpose of this correspondence is to generalize the ECA theory to sampled-data systems, i.e., design discrete-time Kalman filters for a continuous-time system such that the sampled-data estimation covariance can be assigned to a prespecified value. We develop here an equivalent presentstate dependent discrete-time model (see, e.g., [2], [6], and [7]) to obtain the desired sampled-data filters. Note that the dual problem for sampled-data feedback controller design was initially studied in [1] with state covariance assignment and was further investigated for a class of uncertain systems in [11].

In this correspondence, we consider the sampled-data filtering problem by proposing a new performance criterion in terms of the estimation error covariance. An innovation approach to sampled-data filtering is presented. First, the definition of the estimation covariance for a sampled-data system is given, then the sampled-data filtering problem is reduced to the Kalman filter design problem for a fictitious discrete-time system, and finally, an effective method is developed to design discrete-time Kalman filters in such a way that the resulting sampled-data estimation covariance achieves a prescribed value. We derive both the existence conditions and the explicit expression of the desired filters and provide an illustrative numerical example to demonstrate the directness and flexibility of the present design method. The results obtained in the this note are counterparts of the ECA theory for purely continuous- and discrete-time systems [14].

Throughout this correspondence, superscript $T$ denotes matrix transpose, parentheses $(\cdot)$ around an independent variable indicate an analog function of continuous time or the Laplace transform of such a function, whereas square brackets $[\cdot]$ indicate a discrete sequence or the $z$-transform of a sequence. $E\{\cdot\}$ means the expection operator of the argument.

\section{Definitions And PRoblem Formulation}

Consider the following linear time-invariant continuous stochastic system

$$
\dot{x}(t)=A x(t)+w(t)
$$

with continuous-time measurements

$$
y(t)=C x(t)+v(t)
$$

where

$x \quad n$-dimensional state vector;

y $m$-dimensional measured output vector;

$w(t)$ zero mean Gaussian white noise process with covariance $W>0$

$v(t)$ zero mean Gaussian white noise process with covariance $V>0$

where $w(t)$ and $v(t)$ are uncorrelated.

Apparently, one of the most important steps in designing a satisfactory sampled-data filter is to choose a proper discrete-time model 
(DTEM) for the continuous-time system. It should be pointed out that the direct sampling of measurements containing white components is not allowed because the equivalent discrete-time measurement noise would have an unbounded covariance. Therefore, we employ an averaging-type A/D device

$$
y[k]:=\frac{1}{\tau} \int_{(k-1) \tau}^{k \tau} y(t) d t, \quad(k-1) \tau \leq t<k \tau
$$

where $\tau>0$ is the sampling period, and then obtain a present-statedependent DTEM for (1), (2) as follows (see, e.g., [7]):

$$
\begin{aligned}
x[k+1] & =A_{\tau} x[k]+w_{\tau}[k] \\
y[k] & =C_{\tau} x[k]+v_{\tau}[k]
\end{aligned}
$$

where

$$
\begin{aligned}
A_{\tau}:= & e^{A \tau}, \quad C_{\tau}:=\frac{1}{\tau} C \int_{0}^{\tau} e^{A(\xi-\tau)} d \xi \\
w_{\tau}[k]: & \int_{0}^{\tau} e^{A(\tau-\xi)} w(k \tau+\xi) d \xi \\
v_{\tau}[k]:= & \frac{1}{\tau} \int_{0}^{\tau} v((k-1) \tau+\xi) d \xi \\
& -\frac{1}{\tau} \int_{0}^{\tau} \int_{\xi}^{\tau} e^{A(\xi-\tau)} w((k-1) \tau+\eta) d \eta d \xi
\end{aligned}
$$

and $w_{\tau}[k]$ and $v_{\tau}[k]$ are zero mean white noise sequences with

$$
\begin{aligned}
& E\left[\begin{array}{l}
w_{\tau}[k] \\
v_{\tau}[k]
\end{array}\right]\left[\begin{array}{ll}
w_{\tau}^{T}[k] & \left.v_{\tau}^{T}[k]\right]
\end{array}\right. \\
& =\left[\begin{array}{ll}
W_{d 1} & W_{d 3} \\
W_{d 3}^{T} & W_{d 2}
\end{array}\right]:=W_{d}
\end{aligned}
$$

where

$$
\begin{aligned}
W_{d 1} & :=\int_{0}^{\tau} e^{A \xi} W e^{A^{T} \xi} d \xi \\
W_{d 2} & :=\frac{1}{\tau} V+\frac{1}{\tau^{2}} \int_{0}^{\tau} F(\xi) W F^{T}(\xi) d \xi \\
W_{d 3} & :=-\frac{1}{\tau} \int_{0}^{\tau} e^{A(\tau-\xi)} W F^{T}(\xi) d \xi \\
F(\xi) & :=C \int_{0}^{\xi} e^{A(\eta-\xi)} d \eta .
\end{aligned}
$$

The discrete Kalman filter used to reconstruct the state $x(t)$ of (1) is of the form

$$
\hat{x}[k+1]=G \hat{x}[k]+K y[k]
$$

where $G$ and $K$ are filter gains to be designed.

By defining $\varepsilon_{d}[k]:=x[k]-\hat{x}[k]$, we obtain from (3), (4), and (6) that

$$
\begin{aligned}
\varepsilon_{d}[k+1]= & G \varepsilon_{d}[k]+\left(A_{\tau}-G-K C_{\tau}\right) x[k] \\
& +w_{\tau}[k]-K v_{\tau}[k]
\end{aligned}
$$

and subsequently obtain the augmented system of (3) and (7) as follows:

$$
x_{d}[k+1]=\left(A_{d}+B H M\right) x_{d}[k]+(D+B H J) w_{d}[k]
$$

where

$$
\begin{aligned}
& x_{d}[k]:=\left[\begin{array}{c}
x[k] \\
\varepsilon_{d}[k]
\end{array}\right], \quad w_{d}[k]:=\left[\begin{array}{c}
w_{\tau}[k] \\
v_{\tau}[k]
\end{array}\right] \\
& A_{d}:=\left[\begin{array}{ll}
A_{\tau} & 0 \\
A_{\tau} & 0
\end{array}\right], \quad B:=\left[\begin{array}{c}
0 \\
-I
\end{array}\right] \\
& H:=\left[\begin{array}{ll}
G & K
\end{array}\right], \quad M:=\left[\begin{array}{cc}
I & -I \\
C_{\tau} & 0
\end{array}\right] \\
& D:=\left[\begin{array}{ll}
I & 0 \\
I & 0
\end{array}\right], \quad J:=\left[\begin{array}{ll}
0 & 0 \\
0 & I
\end{array}\right]
\end{aligned}
$$

and $w_{d}[k]$ is a zero mean white noise sequence with covariance $W_{d}>$ 0 .

Assumption 1: The white noise sequences $w_{\tau}$ and $v_{\tau}$ have no correlation with $x_{d}$, i.e.,

$$
E\left\{\left[\begin{array}{c}
x\left[k_{1}\right] \\
\varepsilon_{d}\left[k_{1}\right]
\end{array}\right]\left[w_{\tau}^{T}\left[k_{2}\right] q u a d v_{\tau}^{T}\left[k_{2}\right]\right]\right\}=0, \quad \forall k_{1}, k_{2} \in \mathbb{N} .
$$

Noting that the state $x_{d}[k]$ in (8) represents the state behavior at sampling instants of (1) and (7), we now define the sample-time estimation covariance as follows.

Definition 1: The covariance $X_{d}$ of (8) given by

$$
\begin{aligned}
& X_{d}:=\lim _{k \rightarrow \infty} E\left\{x_{d}[k] x_{d}^{T}[k]\right\} \\
& =\lim _{k \rightarrow \infty} E\left[\begin{array}{c}
x[k] \\
\varepsilon_{d}[k]
\end{array}\right]\left[x^{T}[k] \quad \varepsilon_{d}^{T}[k]\right]:=\left[\begin{array}{cc}
X_{d 1} & X_{d 3} \\
X_{d 3}^{T} & X_{d 2}
\end{array}\right]
\end{aligned}
$$

is said to be the sample-time estimation covariance.

Remark 1: Note that the sample-time estimation covariance is a covariance in discrete-time sense and does not take account of the signal at the whole of the intersample but at the sampling instant. Typically, sampled-data systems have been analyzed and designed through their discrete-time behavior, i.e., their behavior at the sampling instants. While a few performance criteria such as stability or deadbeat response can be judged based only on the discrete-time behavior, many others such as disturbance and noise attenuation, and transient properties (overshoot, settling time, etc.), require a closer look at the intersample behavior [1]. Moreover, in the event of filtering for an inherently time-continuous system in terms of a discrete-time "equivalent," the question of sampling is not trivial [8] since the very small sampling period that is naturally required may result in computational difficulties. In the case when it is necessary to preserve the disturbance attenuation and transient properties of the original continuous-time systems and reduce the computational complexity, the signal is sometimes not suitable to be sampled at the Nyquist rate, and therefore, there should come more interest in the behavior during the intersample periods. It is worth mentioning that in recent years, much attention has been paid to the intersample behavior of continuous-time signals rather than the behavior at sampling instant; see, e.g., [4] and references therein for more details.

Remark 2: Assume that there exists sample-time estimation covariance $X_{d}$ for the system (8). Then, under Assumption 1, it is easy to see that $X_{d}$ is the unique positive definite solution of the discrete Lyapunov equation

$$
\begin{aligned}
X_{d}:= & \left(A_{d}+B H M\right) X_{d}\left(A_{d}+B H M\right)^{T} \\
& +(D+B H J) W_{d}(D+B H J)^{T} .
\end{aligned}
$$

Define $\varepsilon_{s}(t):=x(t)-\hat{x}(t)$, where $\hat{x}(t)=\hat{x}[k], k \tau \leq t<(k+$ 1) $\tau$. We are now able to provide the definition of the sampled-data estimation covariance as follows. 
Definition 2: The sampled-data estimation covariance $X_{s}$ is defined as

$$
\begin{aligned}
X_{s} & :=\lim _{k \rightarrow \infty} \frac{1}{\tau} \int_{k \tau}^{(k+1) \tau} E\left\{x_{s}(t) x_{s}^{T}(t)\right\} d t \\
& :=\left[\begin{array}{ll}
X_{s 1} & X_{s 3} \\
X_{s 3}^{T} & X_{s 2}
\end{array}\right], \quad x_{s}(t):=\left[\begin{array}{c}
x(t) \\
\varepsilon_{s}(t)
\end{array}\right] .
\end{aligned}
$$

Remark 3: The sampled-data estimation covariance takes account of intersample behavior and is thus a more exact performance criterion for sampled-data estimation than the sample-time estimation covariance. Next, we need to show that the definition of sampled-data estimation covariance is compatible with the covariances of augmented systems for purely continuous- and discrete-time estimation. For purely continuous estimation [i.e., the system to be estimated and the filter are both continuous, and hence, $x(t)$ and $\varepsilon_{s}(t)$ are both continuous signals], Definition 2 leads to

$$
\begin{aligned}
X_{s} & :=\lim _{k \rightarrow \infty} \frac{1}{\tau} \int_{k \tau}^{(k+1) \tau} E\left\{x_{s}(t) x_{s}^{T}(t)\right\} d t \\
& =\lim _{t \rightarrow \infty} E\left\{x_{s}(t) x_{s}^{T}(t)\right\}
\end{aligned}
$$

and for purely discrete estimation [i.e., the system to be estimated and the filter are both discrete, and hence, $x(t)$ and $\varepsilon_{s}(t)$ are both discrete signals], Definition 2 turns out to be

$$
\begin{aligned}
X_{s} & :=\lim _{k \rightarrow \infty} \frac{1}{\tau} \int_{k \tau}^{(k+1) \tau} E\left\{x_{s}(t) x_{s}^{T}(t)\right\} d t \\
& =\lim _{k \rightarrow \infty} E\left\{x_{s}[k] x_{s}^{T}[k]\right\} .
\end{aligned}
$$

We are now in a position to state the main objective of this note as follows: For the continuous-time system (1) and (2), find all the discrete-time Kalman filters being of the structure (6) such that the sampled-data estimation covariance achieves a prespecified value $X_{s}>0$. We refer to this problem as the sampled-data estimation covariance assignment (SDECA) problem, which is actually the generalization of the problem studied in ECA theory [14], [15].

Remark 4: We briefly discuss the principle on how to prespecify the sampled-data estimation covariance $X_{s}>0$. Note that the 11-block of $X_{s}$ in (13) $\left(X_{s 1}>0\right)$ represents the steady-state state covariance of system (1), which can be calculated in advance since $A$ is Hurwitz. The 22-block of $X_{s}$ in (13) $\left(X_{s 2}>0\right)$ is the steady-state estimation error covariance and is just the item of interest in this note as stated in Section I. $X_{s 2}$ can be prescribed according to the practical performance requirements, and its diagonal elements (i.e., the steady-state estimation error variance) should not be less than the minimal values obtained from the optimal sampled-data filtering theory. The main aim of this correspondence is actually to design sampled-data filters such that the resulting steady-state error covariance is successfully assigned to a prespecified value $X_{s 2}>0$. The 12-block of $X_{s}$ in (13) ( $X_{s 3}$, which makes $X_{s}$ positive definite) is free, and this freedom offers the possibility to consider other desired performance objectives.

\section{SOLUTION TO PROBLEM SDECA}

The following theorem reveals the relationship between the sampled-data estimation covariance $X_{s}$ and the sample-time estimation covariance $X_{d}$.
Theorem 1: Suppose that the sampled-data estimation covariance $X_{s}>0$ exists; then, $X_{s}$ is given by the following expression:

$$
X_{s}=\frac{1}{\tau} \int_{0}^{\tau}\left[C_{s}(u) X_{d} C_{s}^{T}(u)+W_{s}(u)\right] d u
$$

where $X_{d}$ is the sample-time estimation covariance, and

$$
\begin{aligned}
C_{s}(u) & :=\left[\begin{array}{cc}
e^{A u} & 0 \\
e^{A u}-I & I
\end{array}\right] \\
W_{s}(u) & :=\left[\begin{array}{ll}
W_{1}(u) & W_{1}(u) \\
W_{1}(u) & W_{1}(u)
\end{array}\right] \\
W_{1}(u) & :=\int_{0}^{u} e^{A \xi} W e^{A^{T} \xi} d \xi
\end{aligned}
$$

for all $0 \leq u<\tau$.

Proof: For $k \tau \leq t<(k+1) \tau$ and $0 \leq u<\tau$, we have

$$
\begin{aligned}
x_{s}(t)= & {\left[\begin{array}{c}
x(t) \\
\varepsilon_{s}(t)
\end{array}\right]=\left[\begin{array}{c}
x(k \tau+u) \\
x(k \tau+u)-\hat{x}[k]
\end{array}\right] } \\
= & {\left[\begin{array}{cc}
e^{A u} & 0 \\
e^{A u}-I & I
\end{array}\right]\left[\begin{array}{c}
x[k] \\
\varepsilon_{d}[k]
\end{array}\right] } \\
& +\left[\begin{array}{l}
I \\
I
\end{array}\right] \int_{0}^{u} e^{A(u-\xi)} w(k \tau+\xi) d \xi .
\end{aligned}
$$

Observing that $w$ and $x_{d}$ are uncorrelated, we have

$$
\begin{aligned}
& E\left\{x_{s}(k \tau+u) x_{s}^{T}(k \tau+u)\right\} \\
& \quad=C_{s}(u) E\left\{x_{d}[k] x_{d}^{T}[k]\right\} C_{s}^{T}(u)+w_{s}(u)
\end{aligned}
$$

and therefore

$$
\begin{aligned}
X_{s} & =\lim _{k \rightarrow \infty} \frac{1}{\tau} \int_{0}^{\tau} E\left\{x_{s}(k \tau+u) x_{s}^{T}(k \tau+u)\right\} d u \\
& =\frac{1}{\tau} \int_{0}^{\tau}\left\{C_{s}(u) X_{d} C_{s}^{T}(u)+W_{s}(u)\right\} d u .
\end{aligned}
$$

This proves the theorem.

To make the problem SDECA more tractable, we give the following definition.

Definition 3: Consider the discrete Kalman filter (6) with parameters $G$ and $K$. A prespecified $X_{s}>0$ is said to be assignable if there exists a set of matrices $H=[G K]$ such that the sampled-data estimation covariance achieves $X_{s}$.

It follows from Theorem 1 that $C_{s}(u)$ and $W_{s}(u)$ do not depend on $G$ and $K$. Hence, the following theorem offers the conditions for the solvability of the problem SDECA (or the assignability of a prespecified $X_{s}>0$ ).

Theorem 2: Consider the sampled-data system defined in (1)-(6). A prespecified $X_{s}>0$ is assignable if and only if there exist a unique positive definite matrix $X_{d}>0$ and a set of matrices $H=\left[\begin{array}{ll}G & K\end{array}\right]$ that, respectively, meet (14) and (12).

Proof: We only need to show the uniqueness of $X_{d}$ meeting (4), and the rest follows from Theorem 1 and Remark 2 immediately.

Suppose that both $X_{d 1}>0$ and $X_{d 2}>0$ satisfy (14), that is

$$
\begin{aligned}
& X_{s}=\frac{1}{\tau} \int_{0}^{\tau}\left[C_{s}(u) X_{d 1} C_{s}^{T}(u)+W_{s}(u)\right] d u \\
& X_{s}=\frac{1}{\tau} \int_{0}^{\tau}\left[C_{s}(u) X_{d 2} C_{s}^{T}(u)+W_{s}(u)\right] d u
\end{aligned}
$$


and then we have

$$
\frac{1}{\tau} \int_{0}^{\tau} C_{s}(u)\left(X_{d 1}-X_{d 2}\right) C_{s}^{T}(u) d u=0
$$

The definition (15) of $C_{s}(u)$ indicates that $C_{s}(u) \neq 0$, and hence, $X_{d 1}=X_{d 2}$. The proof of Theorem 2 is completed.

Theorem 2 means that the proposed problem SDECA can be divided into the following two sequential problems.

- Problem 1: For a specified sampled-data estimation covariance $X_{s}>0$, find a positive definite matrix $X_{d}>0$ satisfying (14).

- Problem 2: For the positive definite matrix $X_{d}>0$ determined in Problem 1, find a set of matrices $H=[G K]$ satisfying (12).

We first consider Problem 1. Note that (14) can be rearranged as

$$
\int_{0}^{\tau} e^{\hat{A} u} X_{d} e^{\hat{A}^{T} u} d u=\hat{Q}
$$

where

$$
\hat{A}:=\left[\begin{array}{ll}
A & 0 \\
A & 0
\end{array}\right], \quad \hat{Q}:=\tau\left(X_{s}-\int_{0}^{\tau} W_{s}(u) d u\right) .
$$

Since (16) has a similar form with [1, (3.18)], we can calculate $X_{d}$ by using the algorithm proposed in [1].

Next, the following theorem solves Problem 2.

Theorem 3: Assume that $M X_{d} M^{T}+J W_{d} J^{T}>0$. There exists a solution $H$ that solves (12) if and only if $X_{d}>0$ satisfies

$$
\begin{aligned}
R_{d}:= & X_{d}-A_{d} X_{d} A_{d}^{T}-D W_{d} D^{T}+\left(A_{d} X_{d} M^{T}+D W_{d} J^{T}\right) \\
& \cdot\left(M X_{d} M^{T}+J W_{d} J^{T}\right)^{-1}\left(A_{d} X_{d} M^{T}+D W_{d} J^{T}\right)^{T} \\
\geq & 0 \\
& \operatorname{rank}\left[R_{d}\right] \leq n+m \\
& \left(I-B B^{+}\right)\left(X_{d}-A_{d} X_{d} A_{d}^{T}-D W_{d} D^{T}\right) \\
& \cdot\left(I-B B^{+}\right)=0
\end{aligned}
$$

where $B^{+}$denotes the Moore-Penrose inverse of matrix $B$. Furthermore, if conditions (17)-(19) are satisfied, all $H$ solving (12) can be parametrized by

$$
\begin{aligned}
H= & B^{+}\left[L_{d} V_{d}-\left(A_{d} X_{d} M^{T}\right.\right. \\
& \left.\left.+D W_{d} J^{T}\right)\left(\Gamma_{d}^{-1}\right)^{T}\right] \Gamma_{d}^{-1}+\left(I-B B^{+}\right) Z
\end{aligned}
$$

where $Z$ is an arbitrary matrix with appropriate dimension, $L_{d}$ and $\Gamma_{d}$ are, respectively, any matrix factors of $R_{d}$ and $M X_{d} M^{T}+J W_{d} J^{T}$, i.e., $R_{d}:=L_{d} L_{d}^{T}, L_{d} \in R^{2 n \times(n+m)}$

$$
M X_{d} M^{T}+J W_{d} J^{T}:=\Gamma_{d} \Gamma_{d}^{T}
$$

and $V_{d}$ is given by

$$
V_{d}:=V_{d 1}\left[\begin{array}{cc}
I & 0 \\
0 & U_{d}
\end{array}\right] V_{d 2}^{T}
$$

where $V_{d 1}$ and $V_{d 2}$ come from the following singular value decompositions of matrices $\left(I-B B^{+}\right) L_{d}$ and $\left(I-B B^{+}\right)\left(A_{d} X_{d} M^{T}+\right.$ $\left.D W_{d} J^{T}\right) \Gamma_{d}^{-1}$ :

$$
\begin{aligned}
\left(I-B B^{+}\right) L_{d} & =U_{d 1} \Sigma_{d 1} V_{d 1}^{T} \\
\left(I-B B^{+}\right)\left(A_{d} X_{d} M^{T}+D W_{d} J^{T}\right) \Gamma_{d}^{-1} & =U_{d 1} \Sigma_{d 1} V_{d 2}^{T}
\end{aligned}
$$

and $U_{d}$ is an arbitrary orthogonal matrix with proper dimension.

Proof: After a standard algebraic manipulation of (12), the proof of this theorem is completely analogous to the proof of the main results of [5] and is thus omitted.
Remark 5: Note that $B$ is of full column rank, and hence, the last term on the right-hand side of (20) is equal to zero. Consequently, the filter parameters $G$ and $K$ can be obtained by

$$
\begin{aligned}
H & =[G K] \\
& =B^{+}\left[L_{d} V_{d}-\left(A_{d} X_{d} M^{T}+D W_{d} J^{T}\right)\left(\Gamma_{d}^{-1}\right)^{T}\right] \Gamma_{d}^{-1} .
\end{aligned}
$$

Remark 6: It is clear that if the set of desired filters is not empty, it must be very large. We may utilize the freedom (such as the choice of the orthogonal matrix $U_{d}$ ) contained in the filter design to improve other system properties. An interesting problem for future research is how to exploit the freedom to achieve the specified robust and/or reliable constraints on the filtering process.

Remark 7: It is worth pointing out the differences between the SDECA problem addressed in this correspondence and the optimal sampled-data Kalman filtering problem. The goal of the latter problem is to design an optimal filter that minimizes the estimation error variance, and therefore, the resulting optimal filter is usually unique; it seems that there is not much freedom to be used to achieve other performances, such as robustness and the $H_{\infty}$ (disturbance attenuation rejection) requirement. Note that the "reverse problem" of identifying a continuous-time system from measurements was studied in [3]. On the other hand, since the specified variance (the diagonal element of the specified error covariance) constraints may not be minimal but should meet engineering requirements, the addressed SDECA problem in this correspondence is actually a multiobjective design task that often yields nonunique solutions. After assigning to the system a specified error covariance, there remains much freedom that can be used to attempt to directly achieve other desired performance requirements, but the traditional optimal sampled-data Kalman filtering methods maybe lack such an advantage in the case that the filtering performance objectives are expressed explicitly in terms of the steady-state estimation error variance. This implies that the study on the addressed SDECA problem would be suitable to the case when the error variance constraints are not necessary to be minimal, and the design freedom is highly desirable for achieving further performance requirements. In this sense, the results of this paper may complement those of standard optimal sampled-data Kalman filtering.

\section{ILLUSTRATIVE EXAMPLE}

Consider the following system:

$$
\begin{aligned}
\dot{x}(t) & =-1.7329 x(t)+w(t), \\
W & =2 ; y(t)=0.8 x(t)+v(t), \quad V=0.64 .
\end{aligned}
$$

Setting $\tau=0.4$, we have the following DTEM:

$$
x[k+1]=0.5 x[k]+w_{\tau}[k], \quad y[k]=1.1542 x[k]+v_{\tau}[k]
$$

with

$$
W_{d}=\left[\begin{array}{ll}
W_{d 1} & W_{d 3} \\
W_{d 3}^{T} & W_{d 2}
\end{array}\right]=\left[\begin{array}{cc}
0.4328 & -0.8325 \\
-0.8325 & 1.8970
\end{array}\right] .
$$

The Kalman filter to be designed is $\hat{x}[k+1]=G \hat{x}[k]+K y[k]$, and our purpose is to determine the parameters $G$ and $K$ such that the following specified sampled-data estimation covariance:

$$
X_{s}=\left[\begin{array}{ll}
0.5769 & 0.2930 \\
0.2930 & 0.4449
\end{array}\right]
$$

is assigned to the augmented system

$$
x_{d}[k+1]=\left(A_{d}+B H M\right) x_{d}[k]+(D+B H J) w_{d}[k]
$$


where $x_{d}[k]$ and $w_{d}[k]$ are defined in (9), and

$$
\begin{gathered}
A_{d}=\left[\begin{array}{ll}
0.5 & 0 \\
0.5 & 0
\end{array}\right], \quad B=\left[\begin{array}{l}
0 \\
1
\end{array}\right] \\
H=\left[\begin{array}{ll}
G & K
\end{array}\right], \quad M=\left[\begin{array}{cc}
1 & -1 \\
0.8 & 0
\end{array}\right] \\
D=\left[\begin{array}{ll}
1 & 0 \\
1 & 0
\end{array}\right], \quad J=\left[\begin{array}{ll}
0 & 0 \\
0 & 1
\end{array}\right] .
\end{gathered}
$$

We can obtain from Theorem 1 that

$$
X_{s}=\frac{1}{\tau} \int_{0}^{\tau}\left[C_{s}(u) X_{d} C_{s}^{T}(u)+W_{s}(u)\right] d u
$$

where

$$
\begin{aligned}
& C_{s}(u)=\left[\begin{array}{cc}
e^{-1.7329 u} & 0 \\
e^{-1.7329 u}-1 & 1
\end{array}\right] \\
& W_{s}(u)=\left[\begin{array}{ll}
W_{1}(u) & W_{1}(u) \\
W_{1}(u) & W_{1}(u)
\end{array}\right] \\
& W_{1}(u)=0.5771\left(1-e^{-3.4658 u}\right) \text {. }
\end{aligned}
$$

By exploiting the computational algorithm proposed in [1], we have

$$
X_{d}=\left[\begin{array}{ll}
0.5771 & 0.1834 \\
0.1834 & 0.2256
\end{array}\right]
$$

It is easy to verify that $X_{d}>0$ meets all the conditions of Theorem 2, and thus, $X_{s}>0$ is assignable. Next, it follows from Theorem 3 that

$$
\begin{aligned}
R_{d} & =\left[\begin{array}{cc}
0.36032 & -0.03335 \\
-0.03335 & 0.00855
\end{array}\right] \\
L_{d} & =\left[\begin{array}{cc}
0.60026 & 0 \\
-0.03335 & 0.07591
\end{array}\right] \\
\Gamma_{d} & =\left[\begin{array}{cc}
0.62618 & 0.20920 \\
0 & 1.50543
\end{array}\right], \quad V_{d 1}=\left[\begin{array}{ll}
1 & 0 \\
0 & 1
\end{array}\right] \\
V_{d 2}^{T} & =\left[\begin{array}{cc}
0.74612 & -0.66581 \\
-0.66581 & 0.74612
\end{array}\right] .
\end{aligned}
$$

Finally, by selecting the orthogonal "matrix" $U_{d}$ as $U_{d}=1$ and $U_{d}=-1$, respectively, we obtain two desired filters achieving the expected performance as follows:

$$
\begin{aligned}
& \hat{x}[k+1]=0.70072 \hat{x}[k]-0.42504 y[k] \\
& \hat{x}[k+1]=0.86215 \hat{x}[k]-0.37223 y[k] .
\end{aligned}
$$

\section{CONCLUSIONS}

In this correspondence, we have considered the sampled-data estimation problem for continuous-time systems. The definition of sampled-data estimation covariance is proposed by taking account of intersample behavior. The sampled-data estimation covariance assignment problem has been formulated and solved through two steps. The first step converts the sampled-data estimation covariance to the sample time estimation covariance, and the second step is to assign the sample time estimation covariance determined in the first step to a discrete-time system. The existence conditions of the desired filters and the set of these filters have been derived. Finally, we point out that the main subject of future investigation is to utilize the design freedom to meet further performance requirements such as robustness and/or reliability subjected to measurement uncertainties.

\section{ACKNOWLEDGMENT}

The authors would like to thank the associate editor and three anonymous referees for critical reading and comments. The first author is also grateful to Prof. H. Unbehauen of Ruhr-University Bochum for fruitful discussions and to Prof. D. Prätzel-Wolters of University of Kaiserslautern for helpful suggestions.

\section{REFERENCES}

[1] H. Fujioka and S. Hara, "State covariance assignment for sampled-data feedback control," Int. J. Contr., vol. 61, no. 3, pp. 719-737, 1995.

[2] W. M. Haddad, D. S. Bernstein, H.-H. Huang, and Y. Halevi, "Fixed-order sampled-data estimation," Int. J. Contr., vol. 55, no. 1, pp. 129-139, 1992.

[3] R. H. Jones, "Fitting multivariable models to unequally-spaced data," in Times Series Analysis of Irregularly Observed Data, D. F. Findley, Ed. Heiderberg, Germany: Springer, 1984, pp. 158-188.

[4] P. T. Kabamba and S. Hara, "Worst case analysis and design of sampled data systems," IEEE Trans. Automat. Contr., vol. 38, pp. 1337-1357, 1993.

[5] R. E. Skelton and T. Iwasaki, "Liapunov and covariance controllers," Int. J. Contr., vol. 57, no. 3, pp. 519-536, 1993.

[6] W. J. Steinway and J. L. Melsa, "Discrete linear estimatsion for previous stage noise correlation," Automatica, vol. 7, no. 3, pp. 389-391, 1971.

[7] S. Shats and U. Shaked, "Exact discrete-time modeling of linear analogue systems," Int. J. Contr., vol. 49, no. 1, pp. 145-160, 1989.

[8] H. Unbehauen and G. P. Rao, "Continuous-time approaches to system identification-a survey," Automatica, vol. 26, pp. 23-35, 1990.

[9] Z. Wang, Z. Guo, and H. Unbehauen, "Robust $H_{2} / H_{\infty}$-state estimation for discrete-time systems with error variance constraints," IEEE Trans. Automat. Contr., vol. 42, pp. 1431-1435, 1997.

[10] Z. Wang and B. Huang, "Robust $H_{2} / H_{\infty}$ filtering for linear systems with error variance constraints," IEEE Trans. Signal Processing, vol. 48, pp. 2463-2467, Aug. 2000.

[11] Z. Wang, P. Huo, and H. Unbehauen, "Robust constrained covariance controller design for uncertain sampled-data systems," in Proc. Second Asian Contr. Conf., vol. I, Seoul, Korea, July 22-25, 1997, pp. 777-780.

[12] Z. Wang and H. Unbehauen, "Robust $H_{2} / H_{\infty}$-state estimation for systems with error variance constraints: The continuous-time case," IEEE Trans. Automat. Contr., vol. 49, pp. 1061-1465, 1999.

[13] Z. Wang, J. Zhu, and H. Unbehauen, "Robust filter design with timevarying parameter uncertainty and error variance constraints," Int. J. Contr., vol. 72, pp. 30-38, 1999.

[14] E. Yaz and R. E. Skelton, "Continuous and discrete state estimation with error covariance assignment," in Proc. IEEE Conf. Decision Contr., Brighton, U.K., 1991, pp. 3091-3092.

[15] E. Yaz and W. Nacara, "Nonlinear estimation by covariance assignment," in Prep. 12th IFAC World Congr., vol. 6, Sydney, Australia, 1993, pp. 87-90. 